\title{
Routing of internal MANET traffic over external networks ${ }^{1}$
}

\author{
Vinh Pham ${ }^{\mathrm{a}}$, Erlend Larsen ${ }^{\mathrm{a}}$, Øivind Kure ${ }^{\mathrm{b}}$ and Paal Engelstad ${ }^{\mathrm{c}}$ \\ ${ }^{a}$ Unik, Gunnar Randers vei 19, 2007 Kjeller, Norway \\ ${ }^{\mathrm{b}} Q 2 S$, Department of Telematics, NTNU, O.S. Bragstads plass 2a, N-7491 Trondheim, Norway \\ ${ }^{\mathrm{C}}$ Simtel, Simulat/Telernor, P.O.Box 134, 1325 Lysaker, Norway \\ E-mail: \{vph,erl,okure,paalee\}@unik.no
}

\begin{abstract}
Many have proposed to connect Mobile Ad Hoc Networks (MANETs) to a wired backbone Internet access network. This paper demonstrates that a wired backbone network can be utilized for more than just providing access to the global Internet. Traffic between mobile nodes in the ad hoc network may also be routed via this backbone network to achieve higher throughput, and to reduce the load in the ad hoc network. This is referred to as transit routing. This paper proposes a cost metric algorithm that facilitates transit routing for some of the traffic flows between nodes in the MANET. The algorithm aims at carrying out transit routing for a flow only when it leads to improvements of the performance. The proposal is implemented and tested in the ns-2 network simulator, and the simulation results are promising.
\end{abstract}

Keywords: Manet, gateway, cost metric routing, OLSR

\section{Introduction}

Connectivity between Mobile Ad Hoc Networks (MANETs) [1] and the global Internet or other external networks has become an important issue in recent years. Such connectivity increases the usefulness of MANETs in many user scenarios. One example is found in the currently on-going research project, ITEA Easy Wireless [2]. The project proposes to connect a MANET to external networks (such as "Tetra") and the global Internet. This network architecture forms a base communication system for emergency service personnel, governing the fire department, the police department, and emergency medical services. It is also expected that this architecture is applicable to military operation scenarios.

A number of proposals related to Internet connectivity for MANETs have been published lately. Common to many of these proposals is the existence of a wired backbone subnet [3,4], consisting of (i) one or multiple gateways (GWs), and (ii) a number of attached access points (APs) (also called base stations). These nodes are interconnected by high capacity wired links, forming a backbone subnet. Thus there are 3 types of nodes in the network:

\footnotetext{
${ }^{1}$ Based on "Cost Metric Algorithm for Transit Routing in MANETs with Internet Connectivity", by Vinh Pham, E. Larsen, $\emptyset$. Kure and P.E. Engelstad, which appeared in the Proceedings of the 6thInternational Conference on Advances in Mobile Computing and Multimedia, MoMM 2008, November 24-26, 2008, Linz, Austria. (c) 2008 ACM 978-1-60558-269-6/08/0011.
} 
- Gateway nodes are routers that have one or multiple links directly connected to the global Internet or external networks. These nodes are the main entrances into the global Internet or external networks. In frameworks where there is a backbone subnet, these GW nodes are usually equipped with wired interfaces only.

- Access Points nodes are bridges or routers that have both wired and wireless interfaces. They are thus located on the boundary between the wired backbone and the wireless ad hoc subnet. They do not have links directly to the global Internet, but they can reach the Internet through the GWs. Mobile nodes that require Internet access will have their traffic forwarded via one of these APs.

- Mobile nodes are hosts or routers that usually are equipped with one single wireless interface. They are located inside the ad hoc network. While GWs and APs are static nodes, MNs are mobile, and can freely move from one location to another within the MANET.

The primary purpose of a wired backbone subnet is to provide Internet access to mobile nodes (MNs) belonging to an associated mobile ad hoc subnet. Only the traffic between the MANET and the global Internet is routed via the backbone subnet. Traffic between two MNs in the MANET, on the other hand, is routed solely over the wireless links within the MANET.

This paper demonstrates, however, that the wired backbone subnet can also be used for transit routing, meaning that it is used for the communication between two MNs in the MANET. This feature has the potential of improving the overall performance, since the wired backbone subnet is much more reliable and possess higher bandwidth compared to the wireless ad hoc subnet. Other advantages of transit routing are:

- Possibility to achieve higher end to end throughput since the wired backbone subnet has usually much higher bandwidth than wireless links.

- By routing via the wired backbone subnet, the traffic load on the wireless medium may be reduced.

- Greater probability for successful transmissions, since wired links are much more reliable compared to wireless links.

- Wireless communication over many hops is often difficult in ad hoc networks, since the throughput is rapidly decreasing with the number of successive hops. In addition, the probability of unsuccessful transmission is an increasing function with the number of hops. By the aid of the wired backbone subnet, it may be easier to maintain a more stable traffic stream between MNs separated by many hops.

Most MANET routing protocols today, such as OLSR [5], AODV [6], DSR [7] and TBRPF [8] etc., utilize a hop-count metric in the calculation of the routing table. These protocols are commonly referred to as shortest path protocols, and imply that the shortest path (in terms of hops) to a desired destination node is always preferred, no matter what the characteristic of that path is. Hence, transit routing for intranet traffic in our scenario, which in many cases are not the shortest path, is not possible without some modifications to the cost metric used by these protocols.

The proposed optimized cost metric algorithm is designed for this purpose, namely to make transit routing through the backbone subnet possible when appropriate. This means that when there is a performance gain in terms of throughput by using the alternative path through the backbone subnet, the cost metric algorithm will favor this path.

The rest of this paper is organized as follows. In Section 2 we present the work and discussions related to the proposed cost metric routing algorithm. Evaluation results are described in Section 3. Section 4 gives an overview of related works within the area of Internet connectivity in ad-hoc networks. Finally in Section 5 we conclude and point out directions for further work. 


\section{Cost metric routing for intranet traffic}

With the feature of transit routing for intranet traffic introduced in Section 1, higher performance may be achieved in terms of throughput and reduced load in the ad hoc subnet. This is however not always true. There are certainly situations when routing over the wired subnet may result in worse performance. The challenge here is to identify those cases when there is a gain if traffic is routed through the wired backbone subnet, and cases when it is better to just let the traffic be routed along the original path within the ad hoc subnet. This knowledge will be vital for the development of the optimized cost metric algorithm.

\subsection{Reference topology}

In order to obtain an understanding of situations in which it is beneficial or not to favor the alternative transit routing, we have constructed a reference topology as a case study. This topology is shown in Fig. 1, and is mainly based on the works in [3,4]. The difference in our topology and the topologies proposed in these works is the use of multiple gateways. We argue that a reference topology should allow for multiple GWs in the backbone access network, since with more GWs, there will be more bandwidth for Internet traffic. Furthermore, with multiple GWs, traffic may be load balanced between these GWs. Moreover, the network has a higher degree of robustness and reliability because of the redundancy. If one GW encounters failure, there is another GW that can still provide Internet connectivity for MNs in the network. However, these issues are not the main focus of this paper, instead we focus on the derivation of the cost metric routing algorithm that can make transit routing for intranet traffic possible in order to optimize throughput.

The reference topology shown in Fig. 1 consists of two subnets, i.e. (i) the wired backbone subnet and (ii) the wireless ad hoc subnet. GWs and APs are located in the wired subnet, while MNs are located in the ad hoc subnet. As before, the primary task of nodes in the wired backbone subnet is to provide Internet connectivity to MNs in the ad hoc subnet, while the secondary task is to enhance throughput performance for intranet traffic between mobile nodes. Furthermore, the APs, in addition of being access points into the wired backbone subnet, have the important role to extend the access coverage area. One advantage of this extension is that it allows MNs to move over a wider area and still be in the same network. While in the same network, MNs do not need to perform any Mobile IP [9] re-registration (macro mobility), which usually is a very costly process in terms of overhead and handover latency.

In our study, we assume that all nodes in the network, i.e. GWs, APs and MNs, are running the same MANET routing protocol OLSR. The benefit of this choice is that micro mobility within the network is naturally handled by the OSLR routing protocol.

For simplicity of the analysis, we arrange the MNs in the reference topology in a grid formation.

\subsection{Reference throughput}

One key characteristic of multi hop wireless networks is the decreased throughput as the number of hops between source and destination is increased. To verify this, we used the string topology as shown in Fig. 2 to determine the relationship between max throughput and the hop count.

From the simulation result in Fig. 3, we can observe that the throughput is rapidly decreasing when the number of hops $n$ is increasing. For small $n$, the throughput is approximately proportional to $1 / n$, but as $n$ further increases, we see that the throughput is converging to approximately $1 / 5$ of the 1-hop throughput. We can also observe that the reduction in throughput is most severe for the first 2-3 hops. For higher hop counts the reduction in throughput is minimal. The result obtained in Fig. 3 will be used as the reference throughput for the rest of this paper. 


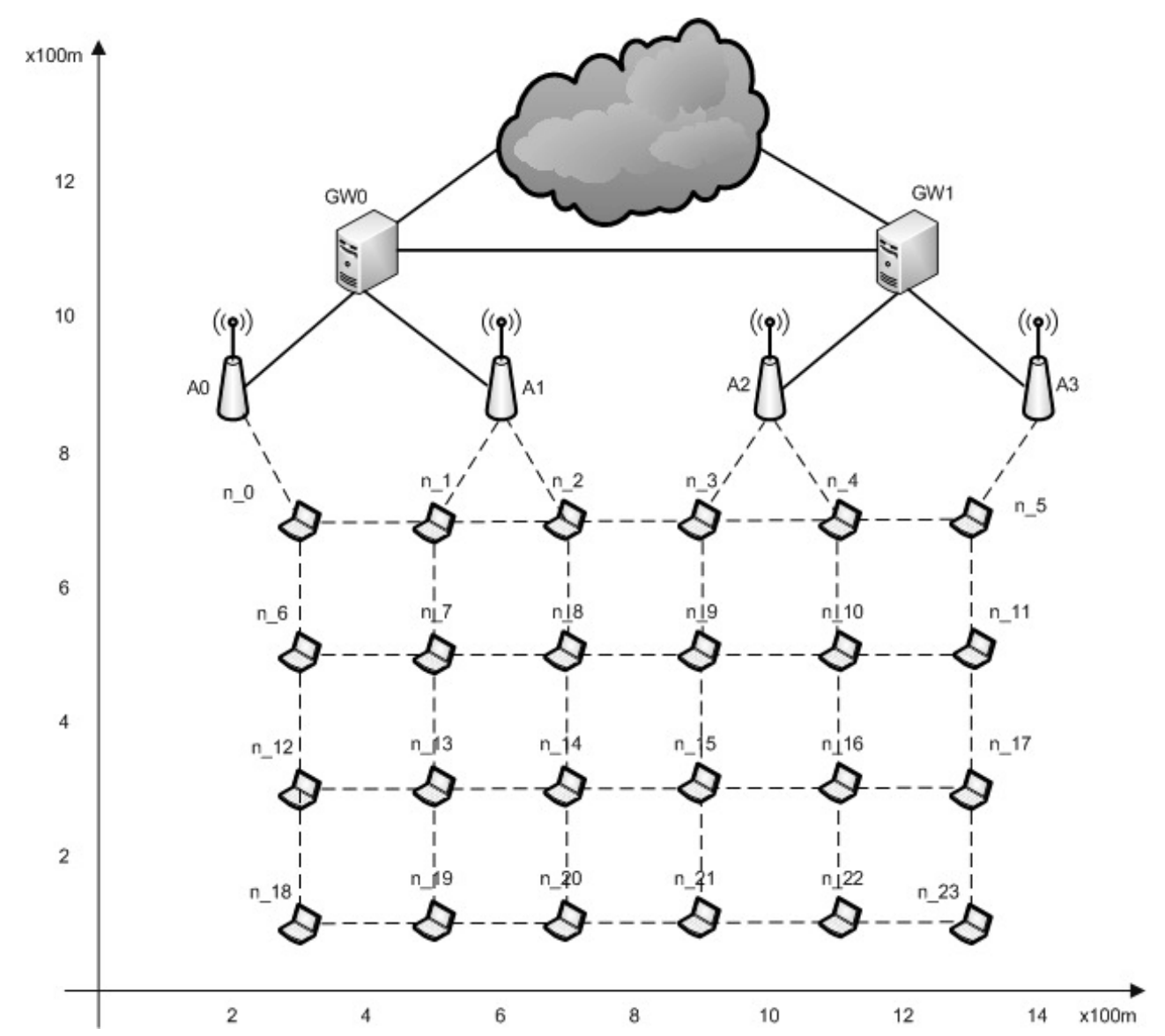

Fig. 1. The reference topology.



Fig. 2. String topology.

\subsection{Enhancement in throughput}

To identify situations in which it is beneficial to perform transit routing, we conducted throughput simulations for all combinations of source and destination pairs from the reference topology. The throughput was simulated for both the wired path and the ad hoc path, and then compared. In Table 1 we list the source and destination pairs in which the simulation result shows a higher throughput for the wired path compared to the ad hoc path. The table also shows the number of wireless hops and the corresponding throughput (kbps) for both the wired path and the ad hoc path.

Here we have for convenience introduced the notation " $m+n$ ", with the understanding that this is a wired path, and it consists of two distinct wireless sections. The first wireless section has $m$ successive wireless hops, while the second wireless section has $n$ successive wireless hops. Since the number of 
Max Throughput vs. Hops

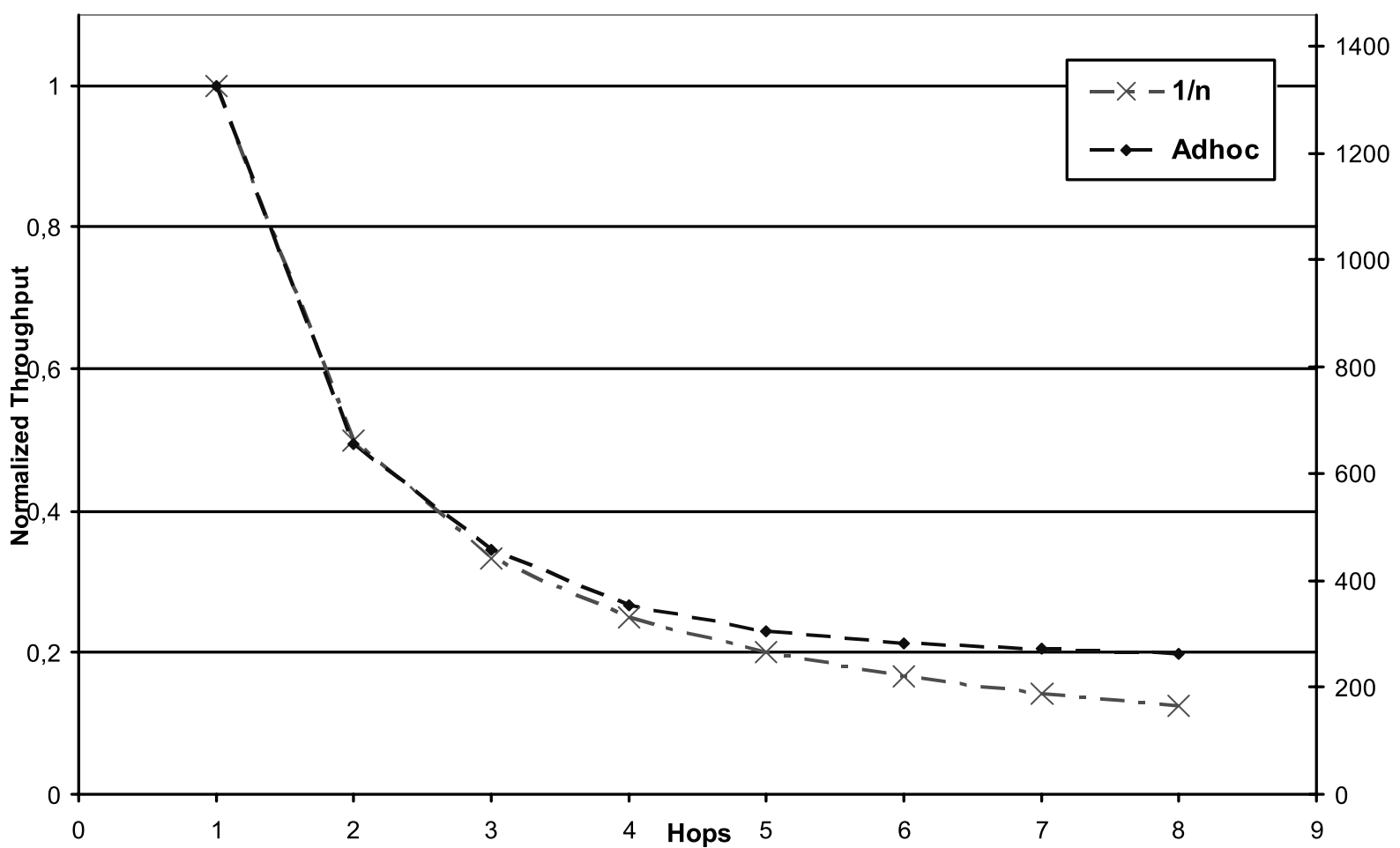

Fig. 3. Reference throughput vs. successive wireless hops.

wired hops has no impact on the throughput in our reference topology, it is therefore omitted in the notation above.

The last column of the table shows the improvements (in percent) in throughput of the wired path compared to the ad hoc path. Moreover, the cell shading code used in the table is as follows: white for cases where the number of wireless hops in the wired path is less than the ad hoc path, light grey where both paths have equal number of wireless hops, and dark grey for cases when the wired path has more wireless hops compared to the ad hoc path.

Due to space limitations, the table only shows a subset of all combinations. However, because of the symmetry in the topology, the remaining combinations of source and destination pairs may be derived from the listed subset.

The result of each entry in Table 1 is an average of 20 simulation runs. Table 2 gives a summary of the parameters used in the simulations. The same parameters are also used in most of the remaining simulations presented in this paper, unless otherwise explicitly stated.

\subsection{Simulation result analysis}

The result from Subsection 2.3 clearly shows that in a number of situations, it is beneficial to apply transit routing for intranet traffic. Furthermore, we can also see that the enhancement in throughput is varying from $16.1 \%$ to $328.4 \%$, depending on the source and destination pair. Now let us define $w$ and $a$ as the total number of wireless hops for the wired path and the ad hoc path, respectively. The average enhancement in throughput is greatest $(109.81 \%)$ for the rows colored white in Table 1, as in these cases, 
Table 1

Source-destination pairs with improved throughput using the wired path.

\begin{tabular}{|c|c|c|c|c|c|c|c|}
\hline$\#$ & Src & Dst & Adhoc & Max Thr. & Wired & Max Thr. Incr. \% \\
\hline 1 & 0 & 3 & 3 & 449.99 & $1+1$ & 1299.30 & 288.7 \\
\hline 2 & 0 & 4 & 4 & 350.87 & $1+1$ & 1299.18 & 270.3 \\
\hline 3 & 0 & 5 & 5 & 302.35 & $1+1$ & 1295.35 & 328.4 \\
\hline 4 & 0 & 9 & 4 & 336.46 & $1+2$ & 637.38 & 89.4 \\
\hline 5 & 0 & 10 & 5 & 291.53 & $1+2$ & 641.50 & 120.1 \\
\hline 6 & 0 & 11 & 6 & 259.16 & $1+2$ & 649.42 & 150.6 \\
\hline 7 & 0 & 15 & 5 & 281.57 & $1+3$ & 447.39 & 58.9 \\
\hline 8 & 0 & 16 & 6 & 254.30 & $1+3$ & 450.33 & 77.1 \\
\hline 9 & 0 & 17 & 7 & 232.39 & $1+3$ & 454.58 & 95.6 \\
\hline 10 & 0 & 21 & 6 & 248.87 & $1+4$ & 348.85 & 40.2 \\
\hline 11 & 0 & 22 & 7 & 231.08 & $1+4$ & 351.08 & 51.9 \\
\hline 12 & 0 & 23 & 8 & 216.51 & $1+4$ & 350.63 & 62.0 \\
\hline 13 & 1 & 4 & 3 & 451.67 & $1+1$ & 644.08 & 42.6 \\
\hline 14 & 1 & 5 & 4 & 350.10 & $1+1$ & 1287.18 & 267.7 \\
\hline 15 & 1 & 10 & 4 & 336.24 & $1+2$ & 450.97 & 34.1 \\
\hline 16 & 1 & 11 & 5 & 292.28 & $1+2$ & 650.08 & 122.4 \\
\hline 17 & 1 & 16 & 5 & 280.37 & $1+3$ & 400.28 & 42.8 \\
\hline 18 & 1 & 17 & 6 & 250.17 & $1+3$ & 454.54 & 81.7 \\
\hline 19 & 1 & 22 & 6 & 246.91 & $1+4$ & 317.74 & 28.7 \\
\hline 20 & 1 & 23 & 7 & 231.57 & $1+4$ & 354.70 & 53.2 \\
\hline 21 & 2 & 5 & 3 & 453.85 & $1+1$ & 1272.60 & 180.4 \\
\hline 22 & 2 & 11 & 4 & 335.89 & $1+2$ & 649.89 & 93.5 \\
\hline 23 & 2 & 17 & 5 & 281.44 & $1+3$ & 452.47 & 60.8 \\
\hline 24 & 2 & 23 & 6 & 248.24 & $1+4$ & 354.88 & 43.0 \\
\hline 25 & 6 & 9 & 3 & 446.05 & $2+2$ & 649.04 & 45.5 \\
\hline 26 & 6 & 10 & 4 & 348.07 & $2+2$ & 651.44 & 87.2 \\
\hline 27 & 6 & 11 & 5 & 299.38 & $2+2$ & 649.53 & 117.0 \\
\hline 28 & 6 & 15 & 4 & 335.80 & $2+3$ & 448.86 & 33.7 \\
\hline 29 & 6 & 16 & 5 & 288.95 & $2+3$ & 450.53 & 55.9 \\
\hline 30 & 6 & 17 & 6 & 258.07 & $2+3$ & 454.28 & 76.0 \\
\hline 31 & 6 & 21 & 5 & 280.79 & $2+4$ & 349.29 & 24.4 \\
\hline 32 & 6 & 22 & 6 & 250.05 & $2+4$ & 352.42 & 40.9 \\
\hline 33 & 6 & 23 & 7 & 230.05 & $2+4$ & 354.28 & 54.0 \\
\hline 34 & 7 & 11 & 4 & 347.53 & $2+2$ & 644.39 & 85.4 \\
\hline 35 & 7 & 17 & 5 & 287.02 & $2+3$ & 455.07 & 58.5 \\
\hline 36 & 7 & 23 & 6 & 249.07 & $2+4$ & 355.32 & 42.7 \\
\hline 37 & 8 & 11 & 3 & 451.82 & $2+2$ & 639.37 & 41.5 \\
\hline 38 & 8 & 17 & 4 & 336.14 & $2+3$ & 452.93 & 34.7 \\
\hline 39 & 8 & 23 & 5 & 283.16 & $2+4$ & 355.31 & 25.5 \\
\hline 40 & 12 & 16 & 4 & 349.60 & $3+3$ & 457.78 & 30.9 \\
\hline 41 & 12 & 17 & 5 & 301.38 & $3+3$ & 457.08 & 51.7 \\
\hline 42 & 12 & 22 & 5 & 290.78 & $3+4$ & 352.80 & 21.3 \\
\hline 43 & 12 & 23 & 6 & 260.80 & $3+4$ & 355.71 & 36.4 \\
\hline 44 & 13 & 17 & 4 & 349.01 & $3+3$ & 454.37 & 30.2 \\
\hline 45 & 13 & 23 & 5 & 289.09 & $3+4$ & 355.90 & 23.1 \\
\hline 46 & 18 & 23 & 5 & 303.37 & $4+4$ & 353.48 & 16.5 \\
\hline & & & & & & & \\
\hline
\end{tabular}

$w<a$. In the cases when $w=a$, i.e. light grey rows, the average enhancement in throughput is $61.77 \%$. The lowest average enhancement in throughput is, as expected, for the case when $w>a$ (dark grey rows). This is summarized in Table 3. From this result we can draw the conclusion that the optimized cost metric algorithm that we shall derive, must as least support transit routing for the cases when $w \leqslant a$. In these cases, the alternative wired path has less or equal number of wireless hops compared to the ad 
Table 2

Simulation parameters

\begin{tabular}{lc}
\hline Simulator & ns-2.31 \\
\hline Routing protocol & UM-OLSR 0.8 .7 \\
OLSR hold time & $20 \mathrm{sec}$ \\
Packet size & $512 \mathrm{MByte}$ \\
Interface queue size & 50 Packets \\
Data rate (wired) & $100 \mathrm{mbps}$ \\
Data rate (wireless) & $2 \mathrm{mbps}$ \\
Data range & $250 \mathrm{~m}$ \\
Carrier sensing range & $550 \mathrm{~m}$ \\
\hline
\end{tabular}

Table 3

Average enhancement in throughput

\begin{tabular}{cccc}
\hline Diff. $w$ and $a$ & $w<a$ & $w=a$ & $w>a$ \\
\hline Avg. enh. thr.put(\%) & 109.81 & 61.77 & 39.95 \\
\hline
\end{tabular}

hoc path. For the cases when $w>a$, the cost metric algorithm may or may not support transit routing, since these cases imply a higher load on the ad hoc subnet.

\subsubsection{Throughput in the case of no interference}

The results from Table 1 also revealed some interesting characteristic regarding throughput vs. interference or no interference. We first investigate the case without interference, that is the case where the first and the second wireless sections of the wired path are not interfering with each other (the distance between them is greater than the carrier sensing range, which by default is $550 \mathrm{~m}$ ). As an example, if we take a look at the throughput for node $\mathrm{n} \_0$ to $\mathrm{n} \_5, \mathrm{n} \_0$ to $\mathrm{n} \_11, \mathrm{n} \_0$ to $\mathrm{n} \_17$ and $\mathrm{n} \_0$ to $\mathrm{n} 23$. These cases have the combinations " $1+1$ ", " $1+2$ ", " $1+3$ " and " $1+4$ ". We take the throughput for these combinations and plot the results together with the reference throughput. The resulting curve is shown in Fig. 4. Here the curve for the combinations above is named " $1+n$ ", since the first wireless section consists of only one wireless hop, and $n$ is the number of wireless hops of the second wireless section. As shown in Fig. 4, the curve " $1+n$ " is almost identical to the reference throughput. This means that the combination " $1+1$ " has a throughput equal to 1 hop of the reference throughput, " $1+2$ " has a throughput equal to 2 hops of the reference throughput, and so on.

If we repeat the same test for the throughput from node $n \_6$ to $n \_5, \mathrm{n} \_6$ to $\mathrm{n} \_11, \mathrm{n} \_6$ to $\mathrm{n} \_17$ and $\mathrm{n} \_6$ to $\mathrm{n} \_23$ and plot the result in the same curve. We now see that " $2+1$ " and " $2+2$ " have a throughput equal to 2 hops in the reference throughput, " $2+3$ " and " $2+4$ " have a throughput equal to 3 and 4 hops, respectively. The result is plotted in the curve " $2+n$ ". The same result can also be observed for the curve " $3+n$ ", i.e. from node n_12 to $\mathrm{n}_{-} 5, \mathrm{n} \_12$ to $\mathrm{n} \_11$, and so on.

With the results we have shown, we can conclude that in a general case " $m+n$ ", where the first wireless section has $m$ hops, and the second wireless section has $n$ hops, and they are not interfering with each other, the throughput is equal to the throughput of $\max (m, n)$ hops. This result is very intuitive, since the throughput is constrained by the section with the highest number of successive wireless hops.

\subsubsection{Throughput in the case with interference}

We repeat the test above for the throughput from $n_{-} 1$ to $n_{-} 4, n_{-} 1$ to $n_{-} 10, n_{-} 1$ to $n_{-} 16$ and $n_{-} 1$ to $\mathrm{n} \_22$. In these cases the nodes in the first wireless section $\left(\mathrm{n} \_1, \mathrm{~A} 1\right)$ are interfering with the nodes in the second wireless section (A2, $\mathrm{n}$-4). As a consequence, the spatial channel reuse is reduced, and hence, the throughput is also reduced compared to the case without interference. This is shown in Fig. 5. The 


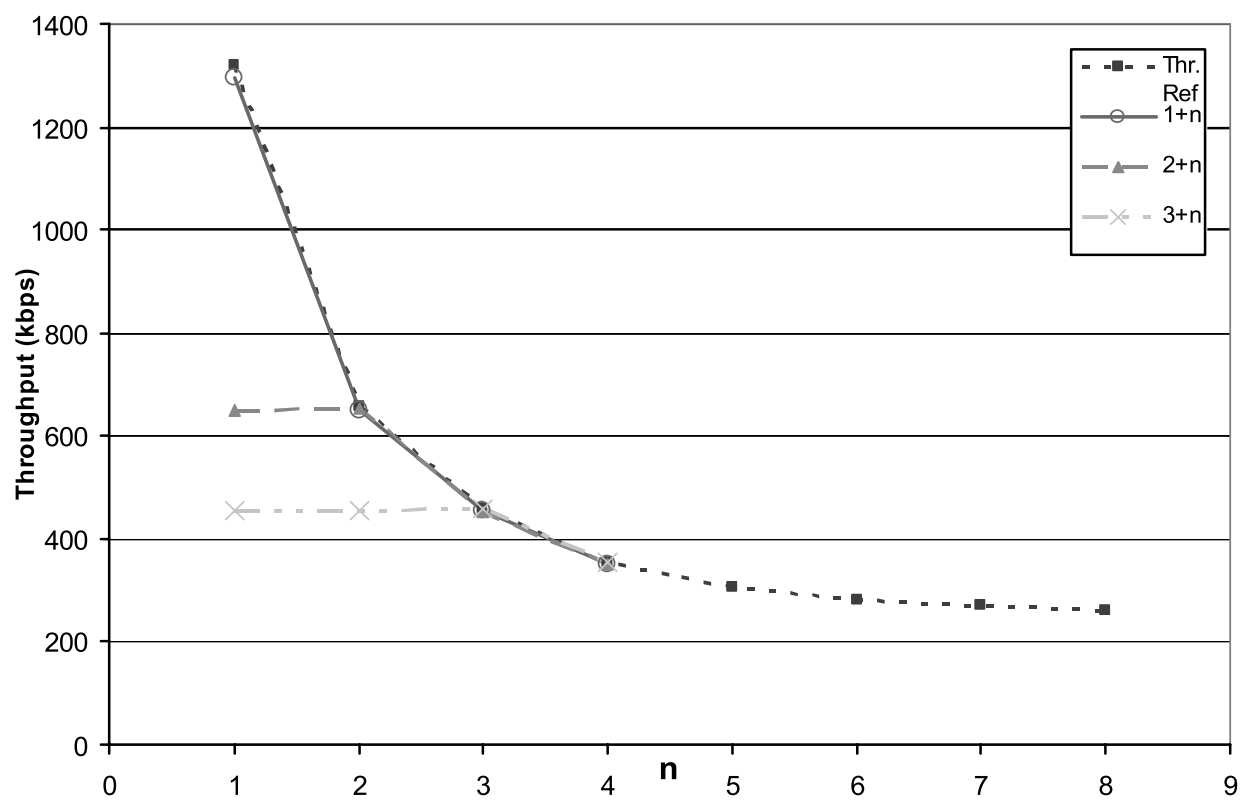

Fig. 4. Throughput with no interference.



Fig. 5. Throughput with interference.

combination " $1+1$ " no longer has a throughput equal to 1 hop as previously, but instead the throughput is now equal to 2 hops in the reference throughput. Likewise, the combination " $1+2$ " has a throughput equal to 3 hops. For the combination " $1+3$ " and " $1+4$ " the throughput is lying between 3 and 4 hops for the first case, and 4 and 5 hops for the latter case. This is because node $\mathrm{n} 16$ and $\mathrm{n} 22$ are not suffered from interference caused by nodes part of the first wireless section. Hence, the throughput from $n \_1$ to 


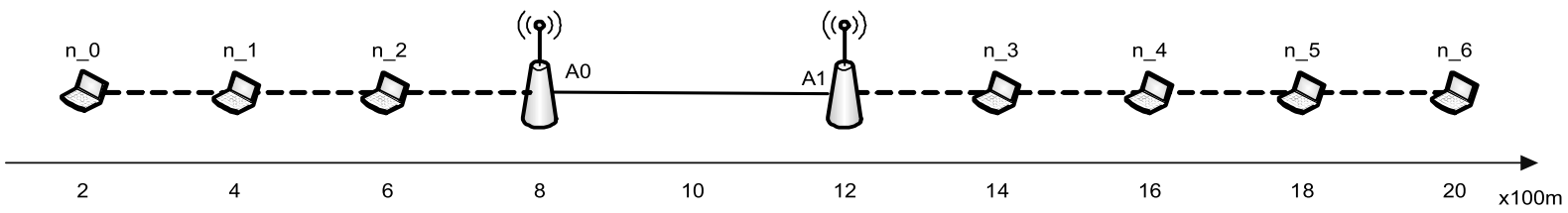

Fig. 6. Throughput with light interference.

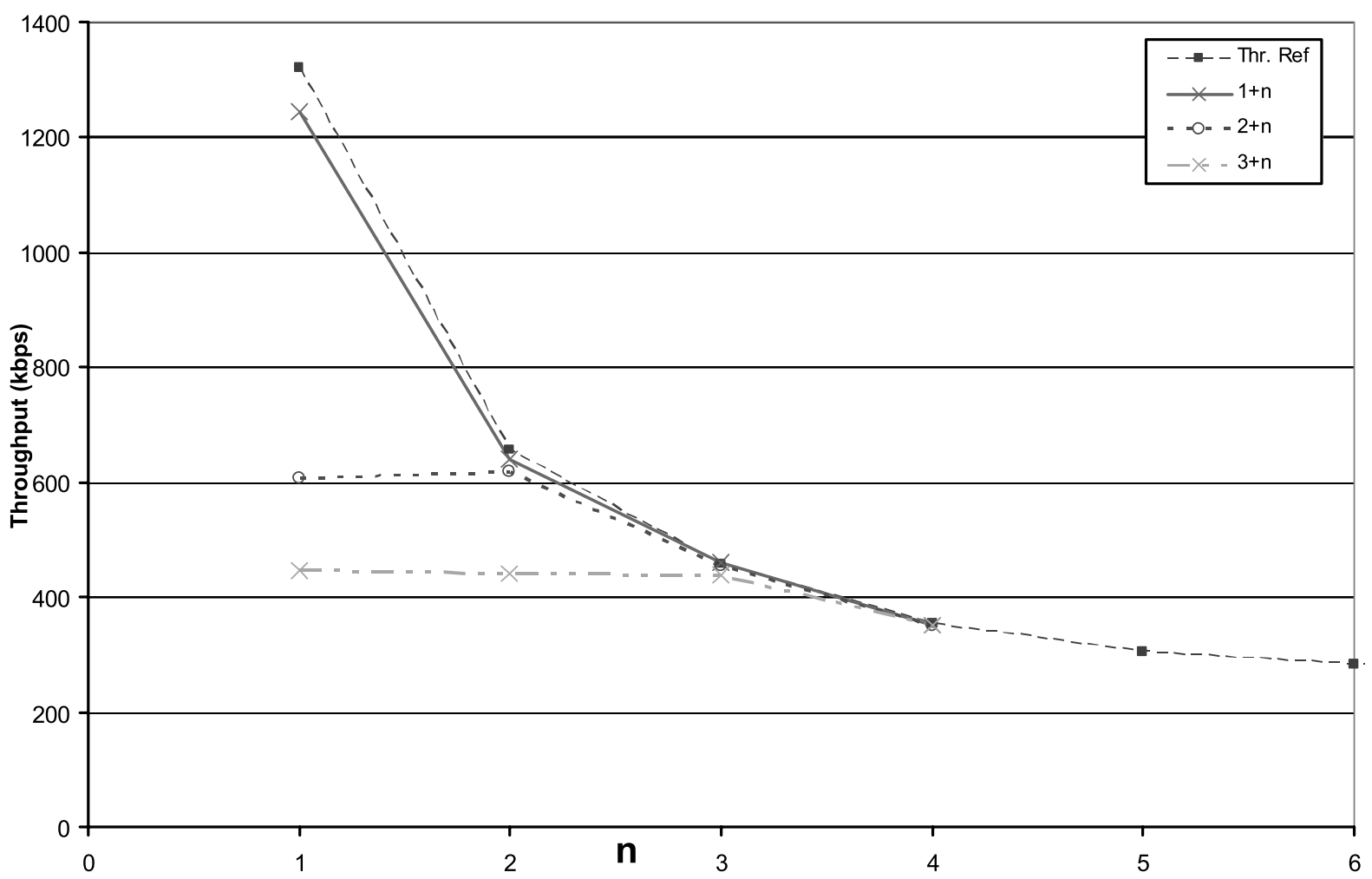

Fig. 7. Throughput with light interference.

$\mathrm{n} \_16$, and $\mathrm{n} \_1$ to $\mathrm{n} \_22$ experience a lighter degree of interference. For the ease of comparison we have also plotted the reference throughput curve transposed one step to the left with the denotation "Thr. Ref. $-1 "$.

To further investigate the impact of interference on the throughput we have constructed two extreme case test scenarios. In the first scenario as shown in Fig. 6, we have arranged the nodes such that the impact of interference is lightest, that is, only the nodes A 0 from the left wireless section, and A1 from the right wireless section are within each others carrier sensing range. The throughput for the combinations " $1+n$ ", " $2+n$ " and " $3+n$ " from left to right is simulated, and the result is shown in Fig. 7. We see that the throughput is approximately equal to the case without interference in Subsection 2.4.1, i.e. the max throughput is constrained by the number of consecutive wireless hops of the longest wireless section. This means that the throughput for a " $m+n$ " combination is equal to the throughput of $\max (m, n)$ hops in the reference throughput.

In the second scenario as shown in Fig. 8a, the nodes are organized such that the impact of interference is most severe. Again we simulate the throughput for the combination " $1+n$ ", " $2+n$ " and " $3+n$ ", 


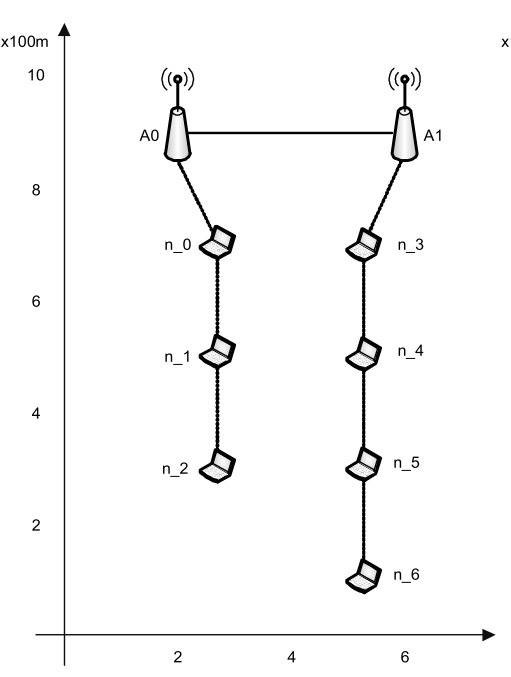

(a)

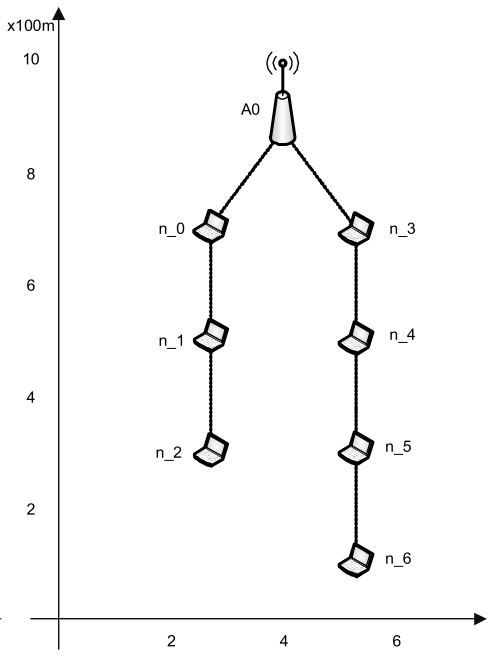

(b)

Fig. 8. Throughput with heavy interference.

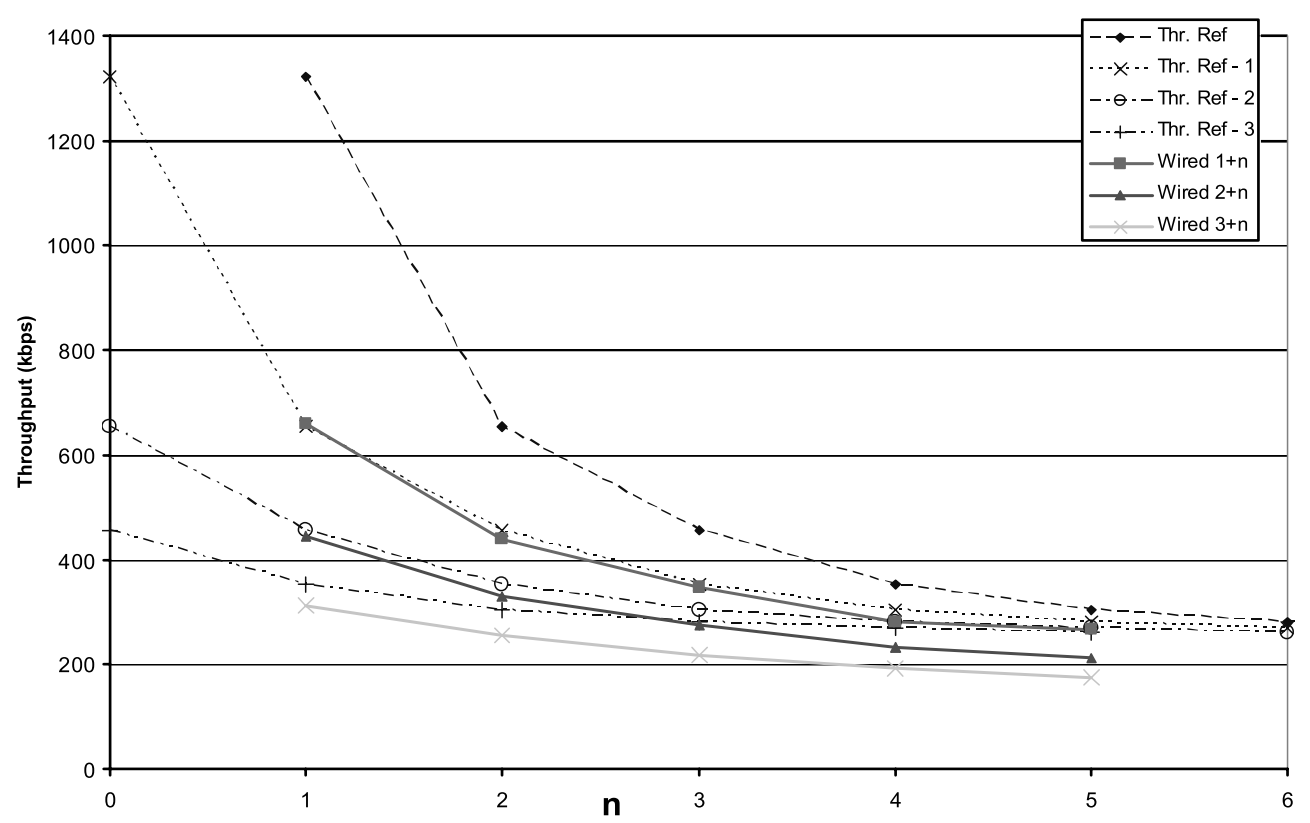

Fig. 9. Throughput with heavy interference.

and the result is shown in Fig. 9. Wee see from this figure that the throughput does no longer have the function $\max (m, n)$ as in the previous case, but instead the throughput is now better described by the function $\operatorname{sum}(m, n)$. This means that the throughput for " $1+1$ " is equal to 2 hops, " $1+2$ " is equal to 3 hops, and so on.

The sum function matches well for the " $1+n$ " curve, but is less accurate in the case " $2+n$ " and " $3+n$ ". This is because we have used the reference throughput obtained from the string topology in 




Fig. 10. Comparison of wired vs. ad hoc path.

Fig. 2 for the comparison, while the topology we are currently investigating is arranged totally different. In order to clear up, we constructed an equivalent topology to the one we are investigating, as shown in Fig. 8b. We simulate the throughput for this equivalent topology in the same way as previously and the result is plotted in Fig. 10. The curves denoted "Ad hoc $1+n$ ", "Ad hoc $2+n$ " and "Ad hoc $3+$ $n$ " are the equivalent throughput to the curves "Wired $1+n$ ", "Wired $2+3 n$ " and "Wired $3+n$ ". As the result shows, the throughput curves for the wired path are almost identical to the corresponding ad hoc path using the topology in Fig. $8 \mathrm{~b}$. This result confirms the correctness of the $\operatorname{sum}(m, n)$ function, i.e. the throughput for the wired path in the case of heavy interference is equal to the throughput of an equivalent ad hoc path with $\operatorname{sum}(m, n)$ wireless hops.

From the results obtained, we may conclude that when the wireless sections " $m+n$ " are interfering with each other, the throughput for the wired path will be equivalent to the throughput of $u$ hops, where $u$ will range from $\max (m, n)$ to $\operatorname{sum}(m, n)$ hops, depending on the degree of interference. In the worst case, the throughput will be equal to the throughput of $\operatorname{sum}(m, n)$ wireless hops.

\subsection{The cost metric routing algorithm}

In this section we will present the derivation of the cost metric algorithms. The cost metric algorithm is a supplement to existing MANET routing protocols such as OLSR, to make transit routing for intranet traffic possible. The aim of this scheme is to optimize the throughput of intranet traffic.

For the discussion below, we use Fig. 11, which is a simplification and generalization of the scenario in Fig. 1. From the figure, MN A is the source which has some data packets to send to MN B. The distance in number of hops from A to the nearest access point to A, say AP a is $m$ hops, while the distance from $\mathrm{B}$ to its nearest access point AP $b$ is $n$ hops. The distance between A and B is $k$ hops through the ad hoc path. Furthermore, the distance from AP $\_$a to AP $\_$b through the backbone subnet is $l$ hops. Node 


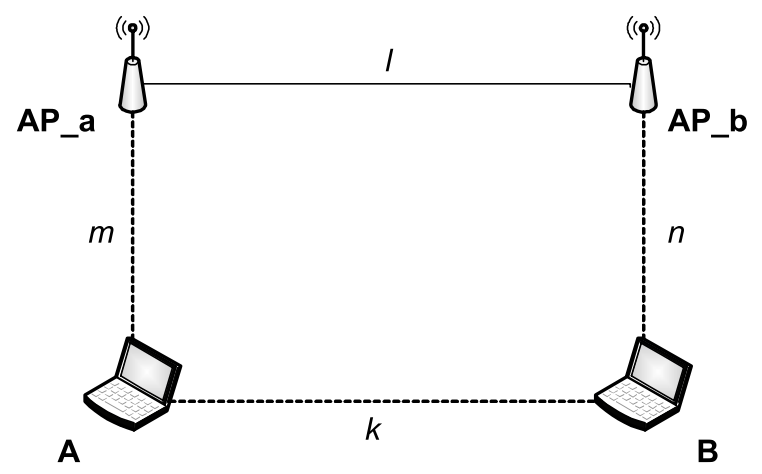

Fig. 11. Generalized scenario for derivation of cost metric routing algorithm.

A can thus send its data packets either through the ad hoc path using $k$ wireless hops, or alternatively it can send the traffic via the wired path, i.e. through the backbone subnet and then to node $\mathrm{B}$, using $m+n$ wireless hops and $l$ wired hops.

The general form of the cost metric algorithm may be written as shown below:

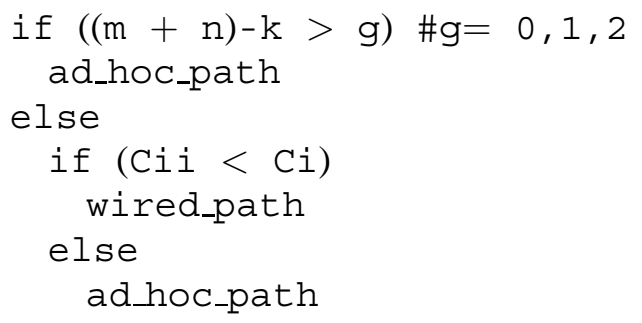

Here, $C_{i}$ and $C_{i i}$ are the total cost for the ad hoc path and the wired path, respectively. The parameter $\mathrm{g}$ is a pre-configurable parameter that determines the "greediness" of the algorithm. For example, if we want to limit transit routing only to cases where " $m+n$ " $\leqslant k+1$, then $\mathrm{g}$ is set to 1 . The purpose of this parameter is to control the accepted amount of extra load on the ad hoc subnet in exchange of a higher throughput using the wired path.

The challenge now is to define the cost function $C_{i}$ and $C_{i i}$ such that $C_{i i}<C_{i}$ for only the cases listed in Table 1 (and also the symmetrical cases that are not listed in the table).

Before we proceed, we define first the cost for wired and wireless hops as follows:

$$
\begin{aligned}
H_{r f} & =\text { Cost for one wireless hop } \\
H_{w} & =\text { Cost for one wired hop. }
\end{aligned}
$$

Here we assume that the cost of one wireless hop is uniform throughout the network. However, the link quality in wireless networks may vary greatly from hop to hop, and hence the cost should ideally reflect this variation. For simplicity and due to the unpredictable nature of wireless networks we find it suitable to utilize a uniform cost for wireless links.

Furthermore, in the derivation of the cost metric algorithm below, we assume that interference can be determined using preconfigured information in the APs. That is, if $A P \_$and $A P b$ are within each others carrier sensing range, then we assume that the wireless sections $m$ and $n$ of the wired path are likely to experience interference. This information must be configured in all APs at the time of deployment. We also assume that this information is made available to all mobile nodes through broadcast messages. 
Now, based on the results in Subsections 2.4.1 and 2.4.2 we define the cost for the ad hoc path with $k$ hops as follows:

$$
C_{i}^{\prime}=k \cdot H_{r f}
$$

Next, we define the cost for the alternative wired path with " $m+n$ " wireless hops. This cost function has two versions, one for the case without interference and one for the case with interference:

$$
\begin{aligned}
& C_{\mathrm{ii}(\text { no interference) }}^{\prime}=l \cdot H_{w}+\max (m, n) \cdot H_{r f} \\
& C_{\mathrm{ii}(\text { interference) })}^{\prime}=l \cdot H_{w}+\operatorname{sum}(m, n) \cdot H_{r f}
\end{aligned}
$$

In the equations above, the parameter $k, l, m$ and $n$ are in accordance with Fig. 6 . We also assume that the values of these parameters are available to the routing protocol through topology information that are periodically disseminated in the network. Moreover, the $\max (m, n)$ in Eq. (3a) is consistent with the result obtained for the case without interference, as discussed earlier in Subsection 2.4.1. On the other hand, the term $\operatorname{sum}(m, n)$ is used in Eq. (3b) for the case with interference, although the result in Subsection 2.4.2 stated that the throughput will be equivalent to the throughput of $u$ successive wireless hops, where $u$ lies somewhere between $\operatorname{sum}(m, n)$ and $\max (m, n)$. Since the degree of interference depends on the location of the wireless nodes (member of the wired path) in relations to each other, and is usually unknown, it is therefore not possible to say with certainty, what the number of hops that the throughput will be equivalent to. However, through our study we have experienced that $\operatorname{sum}(m, n)$ is a better approximation in most cases.

By dividing Eqs (2) and (3) by $H_{r f}$ and by redefining the costs correspondingly, we can rewrite the equations in a simpler form as follows:

$$
\begin{aligned}
& C_{i}=k \\
& C_{\mathrm{ii}(\text { no interference })}=\max (m, n)+c \\
& C_{\mathrm{ii}(\text { interference })}=\operatorname{sum}(m, n)+c
\end{aligned}
$$

where

$$
c=l \cdot \frac{H_{w}}{H_{r f}}
$$

In some scenarios it is natural to assume that communication over a single wired link comes with nearly no cost compared to communication over a single wireless link in a MANET. In this case, one might set $H_{w} / H_{r f}$ in Eq. (6) to an arbitrary small value.

In other scenarios, it is natural to assume that communication over the wired backbone as a whole (e.g. in a small backbone of maximum 10-20 hops) comes with nearly no cost compared to communication over a single wireless link in the MANET. This can be a reasonable assumption, since the throughput in wired links is not dependent on the number of hops in the same way as wireless links (even when the nominal bandwidth of the wired and wireless links are comparable). In this case, one might set $c$ in Eq. (6) such that $c=c^{\prime}$, where $c^{\prime}$ is an arbitrary value between 0 and 1. (Since all other terms in Eqs (4) and (5) are of integer values, the exact value of $c^{\prime}$ will not affect the resulting routing decision).

In this paper, however, we have assumed a scenario where the cost of communication of both wired and wireless links are taken into consideration. In this case, it is natural to associate the cost with the nominal bandwidth over a link, and to assume that the cost is inversely proportional with the nominal 
link bandwidth. Furthermore, we assume that all the wired links have the same nominal bandwidth $B_{w}$ and all the wireless links have the same nominal bandwidth $B_{r f}$. Then, we have:

$$
\begin{aligned}
& H_{r f} \cdot B_{r f}=H_{w} \cdot B_{w} \\
& c=l \cdot \frac{B_{r f}}{B_{w}}
\end{aligned}
$$

The cost metric functions above have been verified to satisfy our requirement of transit routing only for the cases listed in Table 1. This is true as long as $B_{r f}$ is lower than $B_{w}$ such that $c<1$. When $B_{r f}$ increases and hence $c$ increases over this limit we can observe transitions from wired path to ad hoc path for certain combinations of source and destination pairs. To illustrate this we look at the case when the source node is $\mathrm{n}_{-} 1$ and the destination node is $\mathrm{n}_{-} 4$. For this case, $m=1, n=1, k=3$ and $l=$ 3 according to Fig. 1 and Table 1 . The cost for the ad hoc path $C_{i}=3$ while the cost for the wired path $C_{i i}=2+c$. We see that as long as $c<1$, then $C_{i}>C_{i i}$, and the wired path will be preferred, but when $c \geqslant 1$, a transition from wired path to ad hoc path will occur. If the bandwidth of wired links, $B_{w}=100 \mathrm{mbps}$, then $c=3 \cdot B_{r f} / B_{w} \geqslant 1$ when the bandwidth of wireless links, $B_{r f} \geqslant 33.33$ mbps. We also see that this transition is not only dependent on the bandwidth ratio but also on the number of wired hops. In our case $l=3$, but if $l$ is lower then the transition will occur at a higher value of $B_{r f}$, and opposite if $l$ is higher. The example we have shown above is just one incident of a set of transitions. To be more general, the transitions above will occur for all combinations " $m+n$ " with interference, where $\operatorname{sum}(m, n)=k-1$.

Furthermore, a second transition level exists for the case without interference. For example, the cost from node n_0 to node $\mathrm{n}_{-} 3$ along the ad hoc path is $C_{i}=3$, and the cost for the wired path is $C_{i i}=$ $\max (1,1)+c$. We see that when $c<2$ the wired path is preferred, but when $c \geqslant 2$ then the transition to the ad hoc path will occur. If $B_{w}=100 \mathrm{mbps}$ as previously, then the transition will occur when $B_{r f} \geqslant$ 66.67 mbps. This transition level will occur for all combinations " $m+n$ " without interference, where $\max (m, n)=k-2$.

\section{Evaluation}

In order to evaluate the cost metric algorithm described in Section 2, we generated three random topologies, each with 24 mobile nodes as shown in Figs 12-14. For all the test simulations described below, we define a main traffic flow between a source and destination node pair. CBR traffic is transferred between these nodes for a duration of 20 seconds. In each test, the max throughput for this flow is determined. Furthermore, the input data rate of the CBR flow is tuned such that the loss ratio of the flow is less than 5\% of the input rate. Each test is repeated 20 times, and the average max throughput is calculated from the results of these runs. The same test is repeated for a selection of source and destination pairs from all 3 random topologies.

Table 4 summarizes the simulation result for the case of one single data flow without background traffic (BGT). The table is organized as follows: column 1 is the topology of concern. Column 2 and 3 are the source and destination nodes. The parameters $k, l, m$ and $n$ are shown in column 4-7. Column 8 and 9 are the average throughput (in kbps) of the ad hoc path and the wired path, respectively. In column 10, i.e. denoted as Path, the preference of the cost metric algorithm is given. If the wired path is chosen, then the letter $\mathrm{W}$ is used, and if the ad hoc path is chosen then the letter A is used. In the last column, the average enhancement in the throughput of the wired path is given in percentage (\%). 
Table 4

Simulation results 1 traffic flow

\begin{tabular}{crrrrrrrrrr}
\hline Top. & Src & Dst & $m$ & $n$ & $l$ & $k$ & Thr. A & Thr. W & Path & Enh. \% \\
\hline A & 19 & 29 & 2 & 5 & 3 & 8 & 224.78 & 325.86 & W & 44.97 \\
& 21 & 28 & 2 & 2 & 3 & 4 & 338.05 & 650.35 & W & 92.38 \\
& 13 & 21 & 5 & 2 & 3 & 6 & 270.81 & 282.29 & W & 4.24 \\
& 9 & 11 & 1 & 3 & 3 & 4 & 340.24 & 437.86 & W & 28.69 \\
B & 23 & 15 & 2 & 2 & 3 & 4 & 339.16 & 653.71 & W & 92.74 \\
& 22 & 26 & 1 & 4 & 3 & 5 & 287.29 & 341.79 & W & 18.97 \\
& 13 & 21 & 4 & 2 & 3 & 5 & 299.53 & 351.17 & W & 17.24 \\
& 20 & 15 & 6 & 2 & 3 & 7 & 230.51 & 268.32 & W & 16.40 \\
C & 24 & 9 & 3 & 2 & 3 & 5 & 280.79 & 438.97 & W & 56.33 \\
& 22 & 6 & 1 & 3 & 3 & 4 & 350.39 & 436.08 & W & 24.45 \\
& 9 & 15 & 2 & 2 & 3 & 3 & 433.97 & NA & A & NA \\
& 19 & 6 & 3 & 3 & 3 & 6 & 266.65 & 442.78 & W & 66.05 \\
\hline
\end{tabular}

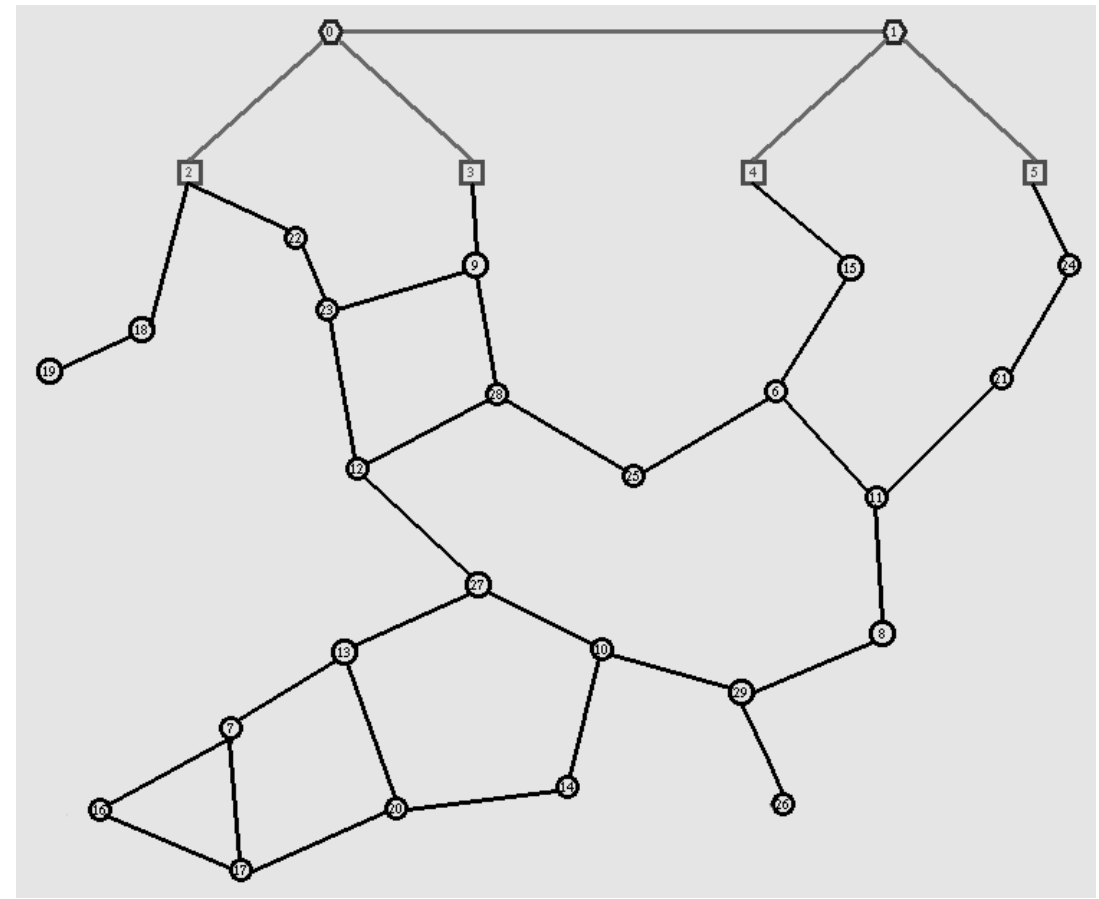

Fig. 12. Random topology A.

All the simulation runs are simulated in the ns-2 [23] simulator, and the "greediness" parameter is configured to be $g=2$. Furthermore, we have chosen to let $c$ in Eqs (5) and (6) to be constant, $c=0.5$. The OLSR Holdtime is set to $20 \mathrm{sec}$ to reduce the potential of route timeouts due to heavy traffic load. By doing so, we can avoid too high variations in the throughput measurements.

Next, the above test is repeated with the presence of BGT. The BGT is emulated by increasing control traffic, i.e. the Hello interval is reduced from 2 to 0.2 seconds. Simulation results are shown in Table 5.

The test is also repeated with 4 BGT flows, each with $100 \mathrm{kbps}$, originating from the 4 APs and destined to dedicated MNs. For Topology A, the BGT flows are defined as follows: from 2 to 27, 3 to 25, 4 to 29 and 5 to 11 . For Topology B, the BGT flows are: 2 to 13,3 to 10, 4 to 26, and 5 to 29 . For Topology C, the BGT flows are: 2 to 12,3 to 27,4 to 14 and 5 to 11 . The results from these simulations 
Table 5

Simulation results Hello $=0.2 \mathrm{sec}$

\begin{tabular}{crrrrrrrrrr}
\hline Top. & Src & Dst & $m$ & $n$ & $l$ & $k$ & Thr. A & Thr. W & Path & Enh. \% \\
\hline A & 19 & 29 & 2 & 5 & 3 & 8 & 203.59 & 301.56 & W & 48.09 \\
& 21 & 28 & 2 & 2 & 3 & 4 & 307.03 & 595.05 & W & 93.81 \\
& 13 & 21 & 5 & 2 & 3 & 6 & 250.79 & 256.12 & W & 2.16 \\
& 9 & 11 & 1 & 3 & 3 & 4 & 313.96 & 411.15 & W & 30.96 \\
B & 23 & 15 & 2 & 2 & 3 & 4 & 309.55 & 605.25 & W & 95.53 \\
& 22 & 26 & 1 & 4 & 3 & 5 & 252.64 & 318.36 & W & 26.01 \\
& 13 & 21 & 4 & 2 & 3 & 5 & 267.42 & 322.08 & W & 20.44 \\
& 20 & 15 & 6 & 2 & 3 & 7 & 210.06 & 249.23 & W & 18.65 \\
C & 24 & 9 & 3 & 2 & 3 & 5 & 260.37 & 411.31 & W & 57.97 \\
& 22 & 6 & 1 & 3 & 3 & 4 & 319.13 & 400.54 & W & 25.51 \\
& 9 & 15 & 2 & 2 & 3 & 3 & 383.68 & NA & A & NA \\
& 19 & 6 & 3 & 3 & 3 & 6 & 245.38 & 408.31 & W & 66.40 \\
\hline
\end{tabular}

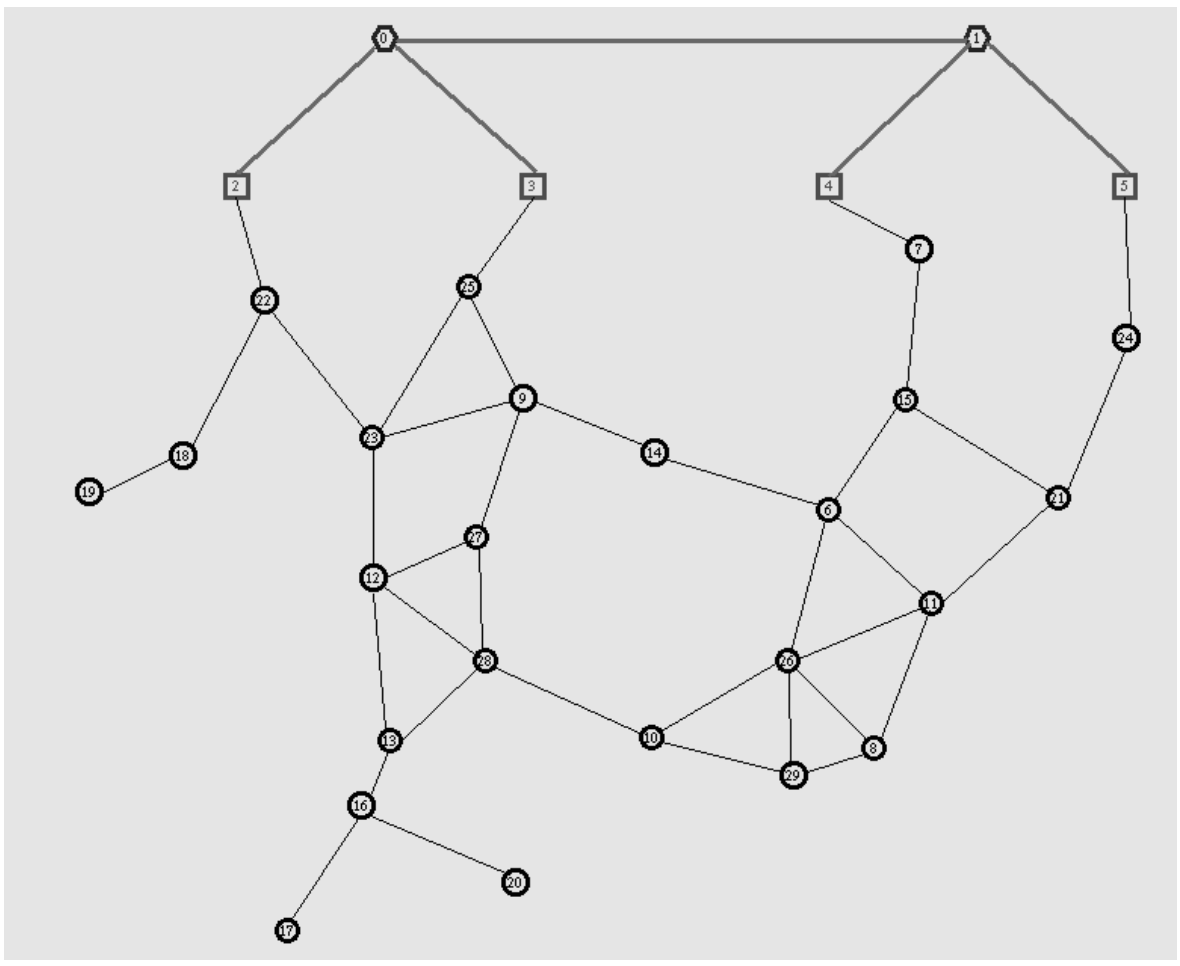

Fig. 13. Random topology B.

are shown in Table 6.

Although the tests are conducted on random topologies, the simulation results validate the correct behavior of the cost metric algorithm. In all the test cases, the algorithm did correctly choose the wired path when it is supposed to. Furthermore, in most cases where the wired path is preferred, we can observe a positive enhancement in the throughput ranging from $2.16 \%$ to $166.94 \%$, and the average throughput enhancement for all test cases is $50.38 \%$. Only in one occasion the wired path resulted in lower throughput compared to the ad hoc path (Topology A, 13 to 21). This occurred in the case with 4 concurrent BGT flows. The reason for the negative enhancement is mainly because of the heavy load in 
Table 6

Simulation results BGT $=100 \mathrm{kbps}$

\begin{tabular}{crrrrrrrrrr}
\hline Top. & Src & Dst & $m$ & $n$ & $l$ & $k$ & $T h r . A$ & Thr. W & Path & Enh. \% \\
\hline A & 19 & 29 & 2 & 5 & 3 & 8 & 97.31 & 171.56 & $\mathrm{~W}$ & 76.30 \\
& 21 & 28 & 2 & 2 & 3 & 4 & 95.46 & 237.62 & $\mathrm{~W}$ & 148.92 \\
& 13 & 21 & 5 & 2 & 3 & 6 & 169.14 & 89.93 & $\mathrm{~W}$ & -46.83 \\
& 9 & 11 & 1 & 3 & 3 & 4 & 112.53 & 230.89 & $\mathrm{~W}$ & 105.18 \\
$\mathrm{~B}$ & 23 & 15 & 2 & 2 & 3 & 4 & 90.81 & 242.41 & $\mathrm{~W}$ & 166.94 \\
& 22 & 26 & 1 & 4 & 3 & 5 & 82.61 & 132.59 & $\mathrm{~W}$ & 60.50 \\
& 13 & 21 & 4 & 2 & 3 & 5 & 87.12 & 101.86 & $\mathrm{~W}$ & 16.92 \\
& 20 & 15 & 6 & 2 & 3 & 7 & 76.48 & 82.69 & $\mathrm{~W}$ & 8.12 \\
$\mathrm{C}$ & 24 & 9 & 3 & 2 & 3 & 5 & 126.76 & 248.21 & $\mathrm{~W}$ & 95.81 \\
& 22 & 6 & 1 & 3 & 3 & 4 & 157.62 & 194.91 & $\mathrm{~W}$ & 23.66 \\
& 9 & 15 & 2 & 2 & 3 & 3 & 160.09 & $\mathrm{NA}$ & $\mathrm{A}$ & $\mathrm{NA}$ \\
& 19 & 6 & 3 & 3 & 3 & 6 & 126.01 & 200.47 & $\mathrm{~W}$ & 59.09 \\
\hline
\end{tabular}

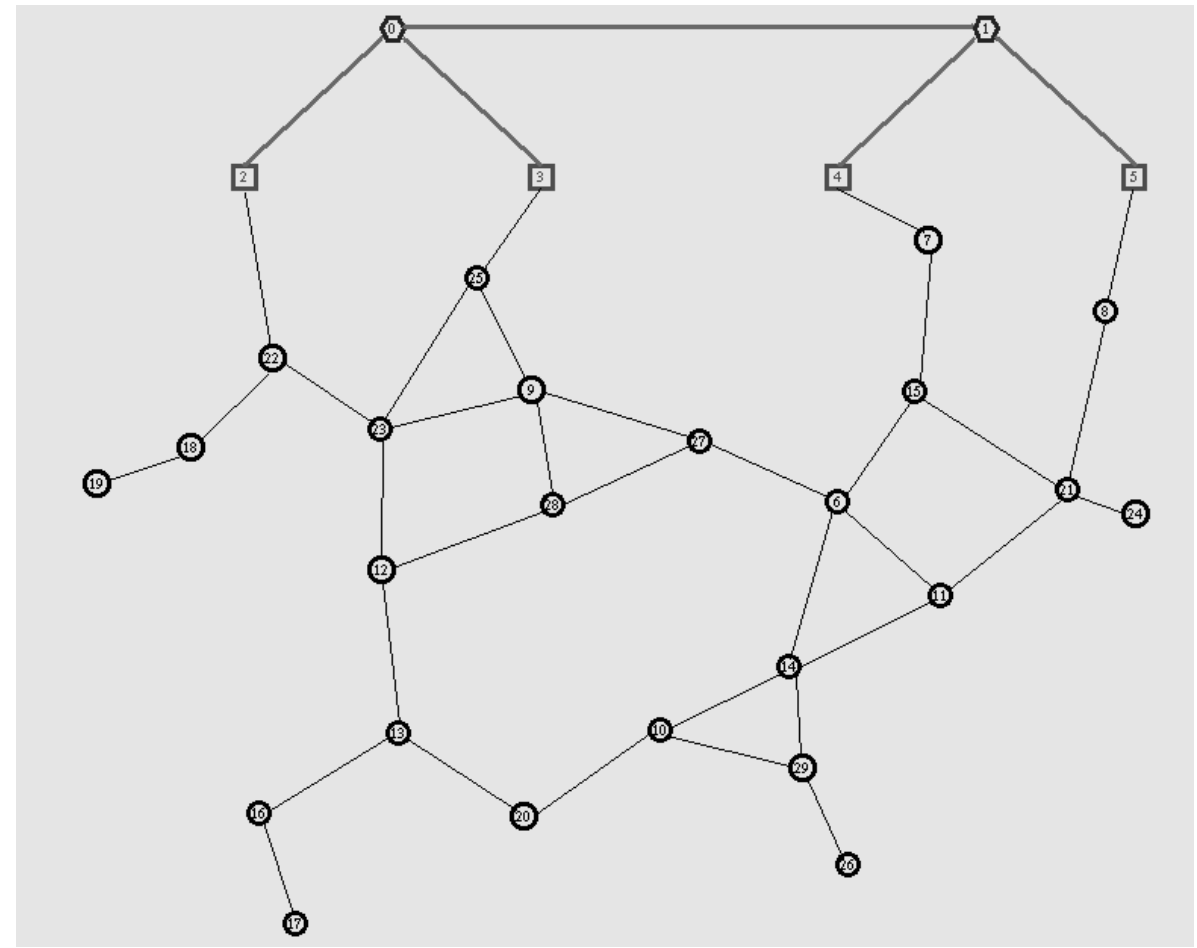

Fig. 14. Random topology C.

the leftmost wireless section, i.e. the path from 13 to 2 or 3 . This load is caused by the BGT flows from 2 to 27 and 3 to 25 which utilize much of the same links as the main traffic flow. In contrast, the ad hoc path from 13 to 21 is not to the same extent affected by the BGT flows. The BGT flows are thus in this particular case in disfavor to the wired path.

Furthermore, we can see from the results that the enhancement in throughput is highest for the cases where $k-\max (m, n) \geqslant 2$, ranging from $44.97 \%$ to $166.94 \%$. For the case when $k-\max (m, n)=1$, the enhancement is moderate, i.e. in average the enhancement is $23.35 \%$.

One interesting result is that in most test cases, the enhancement is increased with increasing back- 


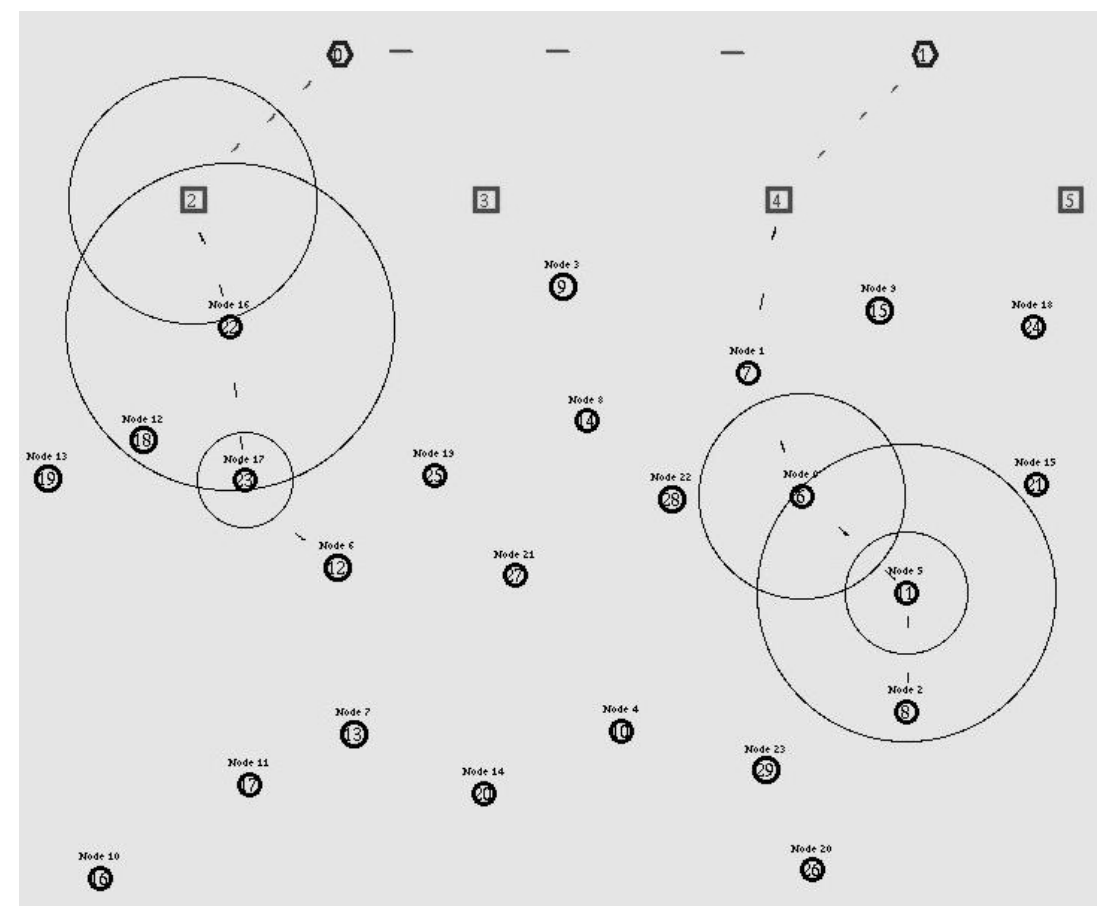

Fig. 15. Snapshot from simulation of topology B. $\mathrm{Src}=12$ and Dest $=8$.

ground traffic load. This is due to the higher number of successive wireless hops of the ad hoc path compared to the wired path, i.e. $k>m$ and $k>n$, combined with the fact that a longer path are more exposed to transmission failure, especially with the presence of BGT.

Finally, we show in Fig. 15 a snapshot from one simulation of topology B. The traffic flow is from the source node 12 to the destination node 8 .

\section{Related works}

There exists a considerable number of papers that are related with Internet connectivity in wireless networks. Some papers [3,4] propose different network architectures to accommodate the internet connectivity requirement. A common approach is to deploy multiple GWs and APs in the network in order to enhance the Internet bandwidth capacity and alleviate the gateway bottleneck problem. However, with the introduction of multiple GWs/APs there are a number of challenges that have to be solved such as gateway forwarding strategies, i.e. tunneling vs. default gateway [10,11].

Other proposals [12-14] are more concerned with how to optimize Internet traffic by applying different load balancing schemes in order to distribute traffic evenly among the gateways that exist in the network. The common solution is to measure the degree of congestion at each gateway utilizing a variety of techniques such as RTT or average queue length [12-15]. If the difference is greater than a threshold value, then the load migration procedure is commenced.

Furthermore, there are a number of works that address the issue of optimizing intranet traffic, commonly referred as multipath routing [16-18]. The idea is to distribute the traffic between two MNs among a set of alternative paths within the ad hoc network in order to achieve higher throughput and minimize the 
impact of local link failure. However, it is reported that the throughput enhancement of this approach is only moderate or negligible, due to the coupling effect in single channel based wireless networks. This fact enforces the importance of transit routing as a mean to enhance intranet traffic performance as well as reducing the traffic load within the ad hoc subnet.

To our knowledge there is only one previous work [19] that is concerned with transit routing. The proposal differs from ours is among other the use of the on demand routing protocol DYMO. Furthermore, the drawbacks with this proposal is that the network is considered static after having been deployed. Otherwise, if a node moves from one associated AP to another closer AP, the node has to acquire a new IP-address with the same prefix as the new AP. This is because the solution relies on the prefix continuity property. Moreover, transit routing is only allowed when it results in a reduction in the number of wireless hops used. They do not consider the property of throughput vs. the number of successive wireless hops. Throughput optimization is therefore not performed e.g. in the case " $2+2$ " vs. 4 hops using the ad-hoc path. Next, the use of an on-demand routing protocol which implies a route discovery latency.

\section{Conclusions/further work}

We have shown that a wired backbone subnet that is normally utilized exclusively for Internet traffic, can also be used for transit routing to enhance the performance of the intranet communication. It will also reduce the traffic load within the ad hoc subnet.

Since most of the current MANET routing protocols utilize a shortest path algorithm with a hop-count based cost metric, transit routing is not possible without modifications to the cost metric. We have proposed a simple and effective cost metric algorithm that can be added to existing routing protocols and thereby making transit routing and higher throughput for intranet traffic possible.

In order to evaluate the proposed solution, we added the cost metric algorithm in the OLSR routing protocol, and performed a number of simulations on 3 random topologies in the ns- 2 simulator. The results from these simulations showed that the cost metric algorithm behaved as intended. Transit routing was enabled only in suitable situations that in most cases resulted in an improved performance in terms of throughput.

Even though the work in this paper is focused on the optimization of the throughput on one single flow of traffic, simulations with background traffic also show on the average considerable enhancement in the throughput. The average enhancement in throughput for all simulations is 50.38\%.

In a more realistic situation, multiple flows of traffic are likely to exist simultaneously in the network. Nonetheless, more realistic traffic model [20] may be used instead of CBR. The task of optimizing the throughput through transit routing will thus be much more challenging in such situations. We intend to address this in follow-on research.

One possible way to solve this is to incorporate some mechanisms for bandwidth monitoring or estimations, such as the ETX [21] or ETT [22]. With the additional information on the traffic load distribution in the network that these extensions can provide, we will be able to derive an improved cost metric algorithm that also accounts for multiple traffic flows. This issue is left to future works.

Although the work in this paper is confined to the throughput optimization of one single traffic flow, the results obtained through this study is expected to be an important foundation for further research and improvements. 


\section{Acknowledgment}

This work was supported by the ITEA Easy Wireless and CELTIC DeHiGate projects.

\section{References}

[1] MANET Working Group of the Internet Engineering Task Force (IETF), homepage, http://www.ietf.org/html.charters/ manet-charter.html

[2] Maurits de Graaf et al., Easy Wireless: broadband ad-hoc networking for emergency services, MedHoc Conference on ad-hoc networking (MedHoc07), 2007.

[3] M. Michalak, Common Gateway Architecture for Mobile Ad-hoc Networks, Second Annual Conference on Wireless On-demand Network Systems and Services (WONS 2005), 2005.

[4] M. Benzaid et al., Integration of Mobile-IP and OLSR for a Universal Mobility, ACM Winet, Wireless Networks Journal 10(4) (July 2004).

[5] T. Clausen and P. Jacquet, Optimized Link State Routing Protocol (OLSR), RFC 3626, October 2003.

[6] C. Perkins et al., Ad Hoc On Demand Distance Vector (AODV) Routing, IETF Internet draft, draft-perkins-manetaodvbis-00.txt, Oct 2003 (Work in Progress).

[7] D. Johnson et al., The Dynamic Source Routing Protocol for Mobile Ad Hoc Networks (DSR) draft-ietf-manet-dsr-09.txt.

[8] R. Ogier et. al., Topology Dissemination Based on Reverse-Path Forwarding (TBRPF), IETF Internet draft, draft-ietfmanet-tbrpd-11.txt, October 2003 (Work in Progress).

[9] C. Perkins, IP Mobility Support for IPv4, RFC 3344, Internet Engineering Task Force (IETF), August 2002.

[10] P.E. Engelstad et al., Internet Connectivity for Multi-Homed Proactive Ad Hoc Networks, Proceedings of IEEE International Conference on Communication (ICC'2004), Paris, June 20-24, 2004.

[11] E. Nordström, et al., Comparison of Gateway Forwarding Strategies in Ad hoc Networks, ACM SIGMOBILE Mobile Computing and Communications Review 2004 (MC2R'04) 8(4) (Oct 2004).

[12] B. Xie et al., Load-balanced mesh router migration for wireless mesh networks, Journal of Parallel and Distributed Computing 68 (6) (June 2008).

[13] K.N. Ramachandran et al., On the Design and Implementatation of Infrastructure Mesh Networks, Proceedings of the IEEE Workshop on Wireless Mesh Networks (WiMesh). IEEE Press (2005).

[14] D. Nandiraju et al., Achieving Load Balancing in Wireless Mesh Networks Through Multiple Gateways, Mobile Adhoc and Sensor Systems (MASS), 2006, Vancouver, Canada.

[15] V. Saigal et al., Load balanced routing in mobile ad hoc networks, Computer Communications 27(3) (15 February 2004).

[16] Mar c Pearlman et al., On the Impact of Alternate Path Routing for Load Balancing in Mobile Ad Hoc Networks, ACM Mobicom, Boston, MA, August 2000.

[17] Y. Ganjali and A. Keshavarzian, Load Balancing in Ad Hoc Networks: Single-path Routing vs. Multi-path Routing, INFOCOM 2004, Twenty-third Annual Joint Conference of the IEEE Computer and Communications Societies.

[18] A. Nasipuri and S.R. Das, On-Demand Multipath Routing for Mobile Ad Hoc Networks, IEEE ICCCN, Boston, MA, October, 1999.

[19] F. Ros and P. Ruiz, A Low Overhead Architecture for Infrastructure-based Wireless Mesh Networks, WiMeshNets 2006, Ontario, August 10, 2006.

[20] A. Doci and F. Xhafa, WIT: A wireless integrated traffic model, Mobile Information Systems 4(3) (August 2008), $219-235$.

[21] D. Couto, A High-Throughput Path Metric for Multi-Hop Wireless Routing, MobiCom '03, San Diego, September 2003.

[22] R. Draves et al., Routing in Multi-radio, Multi-hop Wireless Mesh Networks., ACM MobiCom, Philadelphia, September 2004.

[23] Network Simulator ns-2, http://www.isi.edu/nsnam/ns/.

Vinh Pham (vph@unik.no) received his M.Sc. degree in telecommunication engineering from Norwegian University of Science and Technology (NTNU), Trondheim (1999). He worked a number of years in Telenor and National Defence Research Establishment of Norway (FFI). Currently, he is pursuing his PhD in Ad hoc networks. His research interests include ad hoc and mesh networks, QoS, and routing protocols.

Erlend Larsen (erl@unik.no) received the BE in computer engineering in 1999 and the ME degree in information and communication technology in 2001, both from Agder University College, Grimstad, Norway. From 2001 to 2005 he worked at FFI, the National Defence Research Establishment of Norway, where he is now on a leave of absence. He is currently pursuing 
his PhD with working title Quality of Service mechanisms in Ad Hoc networks" at NTNU, Norway. His research interests include algorithm performance, cross-layer communication and multicast in Mobile Ad Hoc Networks.

Prof. Øivind Kure (okure@ unik.no) is a professor at the Center for Quantifiable Quality of Service in Communication Systems and the Department of Telematics, Norwegian University of Science and Technology, Trondheim. He received his Ph.D. from the University of California, Berkeley in 1988. His current research interest includes various aspects of QoS, performance analysis, multicast protocols and ad hoc networks.

Dr. Paal E. Engelstad (Paal.Engelstad@telenor.com) is associate Professor at UniK, University of Oslo (IFI/UiO), a Senior Research Scientist at Telenor R\&I, and working as a project manager at Simula Research Laboratory. He has published a number of papers over the past years and also holds a number of patents. Paal E. Engelstad has a PhD in Computer Science (UiO 2005), a bachelor in Computer Science (UiO - 2001), a master in Physics (NTNU/Kyoto University, Japan - 1994 - Honors degree with distinction) and a bachelor in Physics (NTNU - 1993) The topic of his PhD was Middleware and Autoconfiguration functionality in Ad Hoc and Personal Area Networks. Nowadays, his main focus is on ad hoc, mesh and wireless sensor networks, performance analysis of IEEE 802.11e, cognitive radio, IMS, IP multihoming, IP mobility, and peer-to-peer overlay networks. 

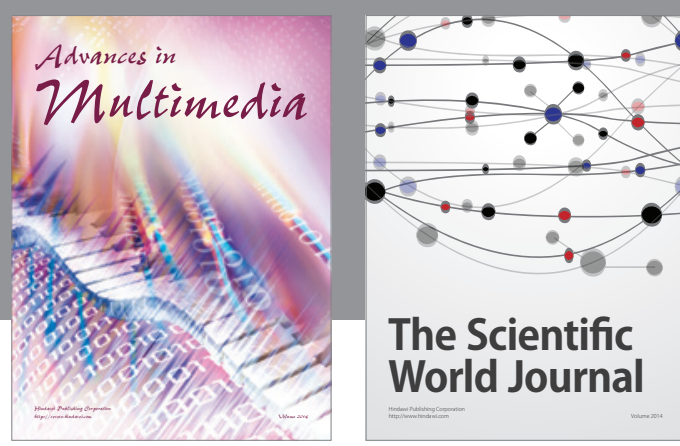

The Scientific World Journal
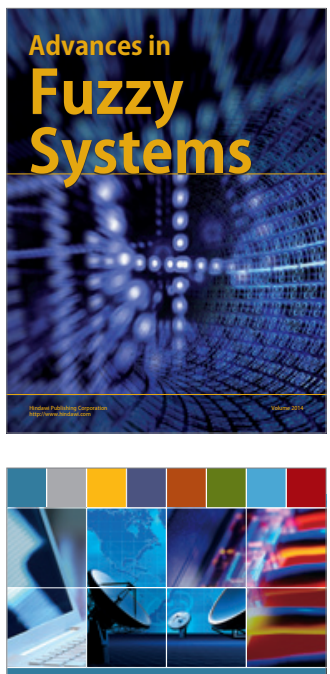

Computer Networks and Communications
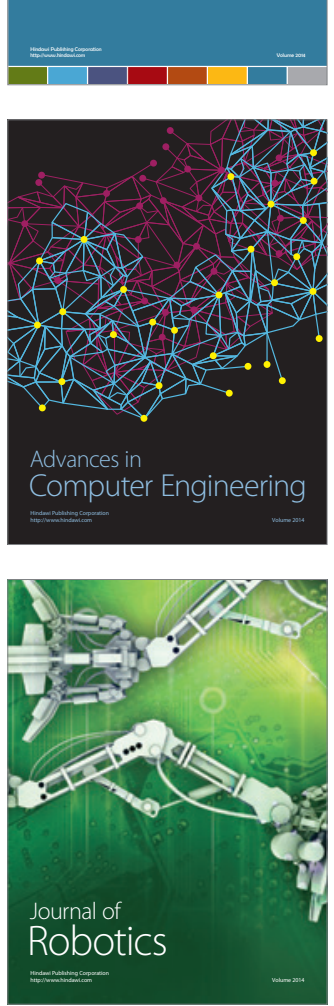
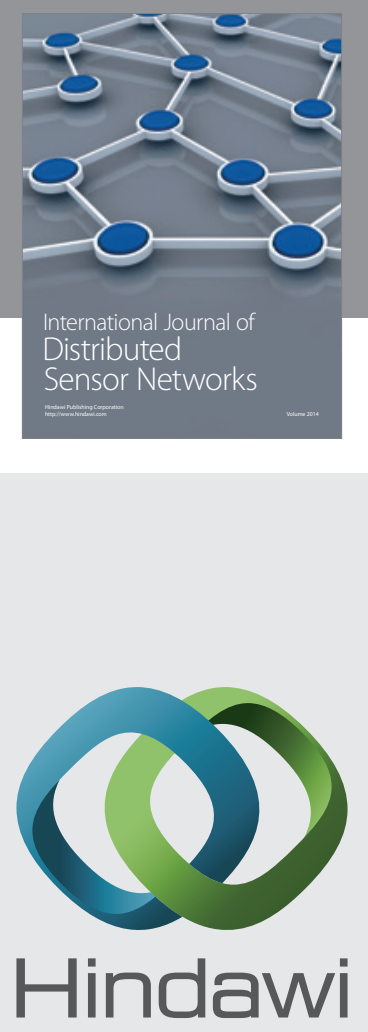

Submit your manuscripts at

http://www.hindawi.com
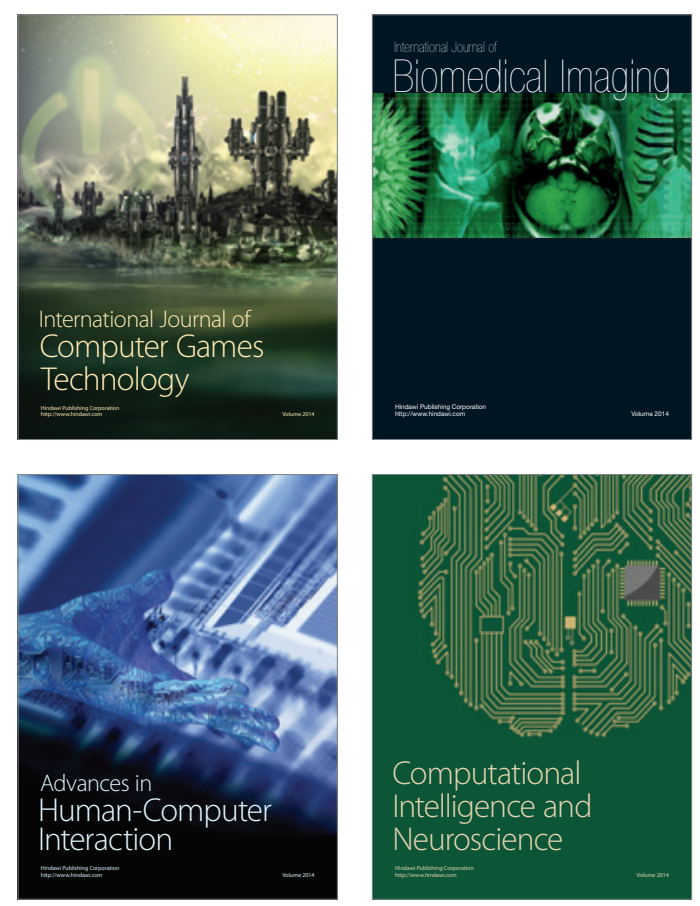
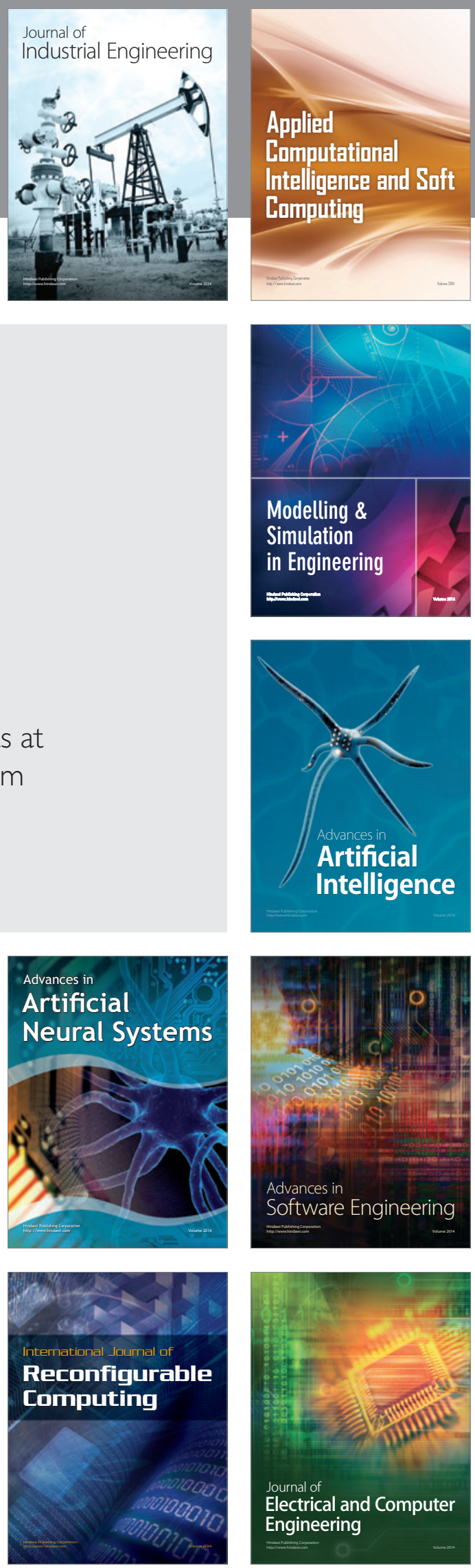